\title{
La valeur épistémologique des langues étrangères dans les Lettres de l'ambassade turque de Milady Montague : le cas du français
}

\section{Rachele Raus}

\section{(2) OpenEdition \\ 1 Journals}

\section{Édition électronique}

URL : https://journals.openedition.org/dhfles/3449

DOI : $10.4000 /$ dhfles.3449

ISSN : 2221-4038

\section{Éditeur}

Société Internationale pour l'Histoire du Français Langue Étrangère ou Seconde

\section{Édition imprimée}

Date de publication : 1 décembre 2012

Pagination : 106-125

ISSN : 0992-7654

\section{Référence électronique}

Rachele Raus, "La valeur épistémologique des langues étrangères dans les Lettres de l'ambassade turque de Milady Montague : le cas du français », Documents pour l'histoire du français langue étrangère ou seconde [En ligne], 49 | 2012, mis en ligne le 04 juillet 2016, consulté le 28 mai 2021. URL : http:// journals.openedition.org/dhfles/3449; DOI : https://doi.org/10.4000/dhfles.3449

Ce document a été généré automatiquement le 28 mai 2021.

\section{(c) SIHFLES}




\title{
La valeur épistémologique des langues étrangères dans les Lettres de l'ambassade turque de Milady Montague : le cas du français
}

\author{
Rachele Raus
}

1 Dans cet article, nous nous proposons d'analyser la valeur épistémologique des langues étrangères, notamment du français, à l'intérieur des Lettres de l'ambassade turque (désormais Lettres turques) ${ }^{1}$ de Lady Mary Montague, aristocrate anglaise partie pour l'Empire ottoman avec son mari Edward en 1716 et arrivée à Constantinople l'année suivante. Notre recherche nous portera à élargir ce corpus de travail à un corpus de référence constitué par la correspondance française de Lady Mary de 1708 à 1720, ainsi que par son ouvrage Discours sur cette maxime de M. La Rochefoucauld: il y a des bons mariages, mais il y en a peu de délicieux, dont la version originale est rédigée en français².

$2 \mathrm{Au} \mathrm{XVII}{ }^{\mathrm{e}}$ siècle, le français se configure désormais comme «langue de prestige » en Angleterre, où pourtant son institutionnalisation se fait également en relation étroite avec d'autres langues (anglais et latin), comme nous le verrons brièvement pour commencer. L'apprentissage que Lady Mary fit des langues étrangères doit être tout d'abord considéré par rapport à ce contexte ainsi qu'en relation avec l'éducation des jeunes femmes au tout début du XVIII ${ }^{\mathrm{e}}$ siècle.

3 C'est après avoir historicisé notre cas d'étude que nous passerons à l'analyse des Lettres turques pour montrer comment à l'intérieur de ce corpus se configure un espace épistémologique complexe où les langues européennes, le français entre autres, s'opposent aux langues orientales, notamment au turc. Enfin, nous aborderons le cas spécifique du français pour voir en quoi cette langue, tout en partageant des valeurs proches des autres langues européennes, finit par nourrir un espace conflictuel qui renvoie à la relation que Lady Mary avait avec cette langue-culture spécifique. 


\section{Quelques prémisses générales : le français en Angleterre au XVII ${ }^{\mathrm{e}}$ siècle}

$4 \quad \mathrm{Au} \mathrm{XVII}^{\mathrm{e}}$ siècle, l'apprentissage des langues en Angleterre doit être considéré d'un côté (approche du haut en bas) par rapport à l'institutionnalisation, voire au « colinguisme » (Branca-Rosoff 2001), et de l'autre (approche du bas en haut) en relation avec la "disciplinarisation" (Suso López 2005). Au niveau de l'institutionnalisation des langues, « at the beginning of the $17^{\text {th }}$ century, French was a requirement primarily for prestige purposes ${ }^{3}$, but latin remained the language of instruction, and English had assumed most official functions " (Kibbee $2001: 180$ ). Cette situation perdure pendant tout le $\mathrm{XVII}^{\mathrm{e}}$ siècle et se renforce au début du siècle suivant, d'autant plus que le latin « perd progressivement son statut de langue véhiculaire en Europe » (Fernàndez Fraile $2012: 53)$.

5 Comme les langues sont perçues à l'époque comme reflétant le « caractère de la nation qui la parle» (Argaud 2009:3), la langue française est d'abord conçue comme langue de prestige parce qu'elle est liée à la mondanité de la cour (Kahane 1986 : 495 ; Fernàndez Fraile 2012 : 52) et au genre romancier. À ce niveau, l'apprentissage via la grammaire et la lecture, notamment par la comparaison entre langues différentes, est indispensable, du moins au niveau théorique. En effet, il s'agit d'acquérir un «savoir savant» (Suso López 2005 : 5), lié à la formalisation d'une langue en train de se faire et qui, pour cela, s'est dotée d'outils de "grammatisation » (Auroux 1993).

6 Si au niveau de l'institutionnalisation des langues, la connaissance et l'utilisation correcte du français sont une marque sociale (Kibbee $1991: 100 ; 2001: 193$; Suso López 2012: 77), au niveau de la disciplinarisation de son enseignement, qui se réalisera définitivement au XIX ${ }^{e}$ siècle, l'apprentissage du français se fait pour des raisons instrumentales (voyage, contact avec les étrangers dans les régions frontalières, commerce, diplomatie). En ce sens, l'accès à un véritable «savoir faire " passe tout d'abord par l'« apprentissage de la langue orale»; par conséquent, "les règles de grammaire n'y sont pas l'élément essentiel, puisqu'on ne parle pas selon des règles » (Suso López 2005 : 5).

7 Nous avons donc au moins deux perceptions de la langue française de cette période : la première qui renvoie à la culture des salons, à laquelle l'apprenant - notamment aristocrate - se forme par des tuteurs ou par le célèbre « Grand Tour »; la deuxième qui est instrumentale.

\section{La formation de Lady Mary dans les langues étrangères}

8 La formation de Mary Pierrepont ${ }^{4}$ dans les langues étrangères débute à l'âge de 13 ans par l'étude du latin, comme elle dit à plusieurs reprises à Joseph Spence (Halsband $1965: 6$, n. 2 ; Grundy 1999 : 15). Cette langue, considérée comme langue « de l'instruction ", était en fait rarement enseignée aux femmes 5 , voilà pourquoi Lady Mary l'apprend au début en autodidacte, comme elle avoue dans son roman autobiographique inédit (Paston 1907: 4). Cela dit, elle jouira bientôt de l'aide de Gilbert Burnet, évêque de Salisbury ${ }^{6}$, qui lui fera traduire l'Enchiridion d'Épictète et qui lui précisera à ce sujet ce qui suit: 
The common opinion is that the Latin language is not suitable for the ladies because it does too little toward the preservation of their modesty, just because it is rare and unusual thing for a woman to know Latin [...] It is proper for a woman born in Germany to learn French so that she can talk with those who know French. Why then is it considered indecorous if she learns Latin so that she can converse daily with so many eloquent, learned and wise authors and trustworthy counselors? (Halsband $1965: 46, \mathrm{n} .1$ )

9 Pour la formation des femmes, aristocrates ou non, l'apprentissage de la langue latine n'était donc pas prévu. Au contraire, celui des langues frontalières était perçu comme licite à des fins instrumentales.

10 Lady Mary se plaint plusieurs fois de sa formation, dénonçant «the silly prejudices of my education » dans une lettre adressée à son fils le 23 juin 1753. À propos de sa formation, Grundy précise :

Masters were employed to instruct Lady Mary in drawing [...] in the Italian language, and in carving joints of meat [...] She also acquired female skills such as embroidery and cooking, and the physical arts of dancing and riding. But the centre of her education was an achievement all her own (Grundy $1999: 20$ ).

11 La formation à l'italien et au français était normalement prévue pour les aristocrates, comme dit Lady Mary dans l'une de ses lettres turques, en faisant une comparaison entre le multilinguisme de l'Empire ottoman et les connaissances des langues des Anglaises :

This [le multilinguisme turc] seems almost incredible to you, and is, in my mind, one of the most curious things in this country, and takes off very much from the merit of our ladies, who set up for such extraordinary geniuses, upon the credit of some superficial knowledge of French and Italian (1799: 230).

12 La critique de Lady Mary confirme ce que Kibbee remarque justement au sujet de l'apprentissage des langues en Angleterre au XVII siècle : "knowledge of foreign languages, particularly French, was a mark of class, but the knowledge apparently did not have to be very profound » (Kibbee 1991 : 101).

L'apprentissage de l'italien date de 1710 , d'après le témoignage que Lady Mary nous fournit dans une lettre adressée à Frances Hewett, l'une de ses correspondantes de jeunesse (Halsband 1965 : 21). Elle doit son éducation italienne à Ludovico Casotti, qui l'aidera plus tard en tant que médiateur pour le mariage avec Edward Wortley (Halsband 1965 : 139).

14 L'apprentissage du français commence plus tôt et Lady Mary devient experte de la langue au point non seulement de traduire aisément du français vers sa langue maternelle mais d'écrire directement dans cette langue son célèbre Discours sur cette maxime de M. La Rochefoucauld (Montague $1768: 61 \mathrm{sv}$ ). En effet, elle possède les qualités nécessaires pour le faire, notamment «fluency in French » et " an understanding of English and French culture and society » (Long 2009).

15 En 1715, elle commence à étudier l'allemand «to make her court to the King » (Halsband 1965 : 245, n. 1). Un an après, elle entreprend son célèbre voyage pour l'Empire ottoman à côté de son mari, chargé de la mission anglaise à la Porte ottomane, ce qui lui permettra d'achever ses études des langues étrangères par l'apprentissage du turc.

16 Précisons que Lady Mary apprend le latin et les langues européennes (italien, français, allemand) d'abord par la grammaire, et ensuite par la lecture de textes en langue 
étrangère. À cet égard, voici ce qu'elle précise à sa future belle-sœur Anne Wortley en 1709 (Halsband $1965: 6$ ) :

My study at present is nothing but dictionnaries and grammars. I'am trying whether it be possible to learn without a master ; I am not certain (and dare hardly hope) I shall make any great progress; but I find the study so diverting, I am not only easy, but pleased with the solitude that indulges it.

Cette approche à l'apprentissage par les outils de grammatisation est par ailleurs mise en doute par sa correspondante :

[...] with out 'em [Anne Wortley se réfère aux règles de grammaire] you would read with pleasure in 2 or 3 months, but if you persisted in the use of 'em you would throw away your Latin after a year or 2 and the Commonwealth of Letters have reason to mourn, whereas if I could prevail with you it would be bound to thank me for a brighter ornament than any it can boast of (ibid. : 9).

Quant aux lectures diverses, la bibliothèque de Lady Mary contient, entre autres, le livre italien Prediche morali (Halsband : 21, n. 3), ainsi que plusieurs romans français, ses deux auteurs préférés étant d'Urfé et Scudéry (Paston 1907 : 4). Ses lectures en français se feront de plus en plus nombreuses au fil des années grâce également aux échanges de livres avec Frances Hewett (Halsband 1965 : 15).

\title{
3. Langues européennes versus langues orientales dans les Lettres turques
}

\author{
Elève: Monsieur (mon Maistre) la langue française est-elle bien utile \& profitable \\ parmi la Noblesse? \\ Maistre : Voire : \& encore plus utile parmi les marchands : car exceptant la latine, (il \\ me semble) qu'elle est la plus utile de la Chrestieneté : \& elle est quasiment autant \\ en usage (si j'osoye dire) que la latine, ou bien plus (Wodroephe $1623: 213$, cité dans \\ Kibbee $2001: 182$ )
}

Cette citation de Wodroephe (1623) permet d'introduire la première remarque que nous pouvons faire à propos des Lettres turques de Lady Mary, à savoir que la perception de la valeur des langues se fonde tout d'abord sur l'opposition chrétien-musulman (nous-l'autre). En effet, le monde européen est conçu tout d'abord comme monde de la chrétienté, notamment de l'aristocratie chrétienne. Quand Lady Mary rectifie la manière dont les voyageurs qui l'ont précédée ont décrit le référent turc, le «nous » qu'elle utilise ne se réfère pas qu'aux Anglais ${ }^{7}$, mais entend l'ensemble de la chrétienté :

[Lettre XXVIII à l'Abbé Conti] 'Tis certain we have but very imperfect accounts of the manners and religion of these people (ibid. : 111).

[Lettre XXIX à la contesse de Mar] extreme stupidity of all the writers that have given accounts of them. 'Tis very easy to see, they [les femmes turques] have in reality more liberty than we have (ibid. : 134).

Lettre XXXII à Mme Thistelthwayte] I suppose you have read, in most of our accounts of Turkey, that their houses are most miserable pieces [...] I assure you, 'tis no such things (ibid. : 160-161).

[Lettre XLII à la contesse de Bristol] Tis also very pleasant to observe how tenderly he [elle se réfère au voyageur anglais Aaron Hilly] and all his brethren voyagewriters lament the miserable confinement of the Turkish ladies [...] you may believe me, that the Turkish ladies have, at least, as much wit and civility, nay liverty, as among us (ibid. : 244-246).

Le renvoi à la tradition des voyageurs européens, qui ont bâti une mémoire discursive spécifique sur le référent ottoman depuis plusieurs siècles (Raus 2006), permet au 
"nous» de se constituer en relation avec l'autre. Remarquons que de cette communauté discursive font partie autant les hommes (Conti) que les femmes (Mar, Thistelthwayte, Bristol), indépendamment de leur nationalité et de leur langue ${ }^{8}$. La relation inverse de l'Ottoman par rapport à l'autre, européen et chrétien, est également présente dans les lettres, comme l'on peut voir à travers le jugement que la dame turque Fatima porte sur les Européennes : "Your Christian ladies (said she, with a smile that made her as beautiful as an angel) have the reputation of inconstancy" (Montagu 1799 : 222).

21 Le renvoi à la chrétienté est fréquent non seulement quand Lady Mary se réfère à ellemême et à l'Ambassadrice de France comme "two young Christian Ambassadresses " (ibid. : 128), mais aussi quand elle souligne le fait d'avoir eu, la première, la possibilité de visiter un harem turc et de s'entretenir avec des dames turques, dont elle a désormais appris la langue. Elle parle à ce sujet $d^{\prime}$ '« extraordinary occasion, that a Christian is admitted into the house of a man of quality [y compris de son harem] " (ibid. : 164), ainsi que d'un « entertainement [avec les dames turques du harem], which was never before given to any Christian » (ibid. : 166).

En tant que langue de l'autre, oriental et musulman, le turc n'a pas la fonction instrumentale de permettre l'échange, mais il acquiert la valeur épistémologique de découverte de l'altérité. En cela, il se rapproche des deux autres langues orientales dont Lady Mary fera l'expérience lors de son voyage : l'arabe et le persan. À ce sujet, la rencontre avec le pacha Achmet à Belgrade se révèle fondamentale : l'utilisation des interprètes lui permet de dépasser la seule approche instrumentale de ces langues et de désirer accéder à la connaissance de l'autre par la langue.

You cannot imagine how much he is delighted with the liberty of conversing with him [...] He has explained to me many pieces of Arabian poetry [...] Their expressions of love are very passionate and lively. I am so much pleased with them. I really believe I should learn to read Arabic, if I was to stay here a few months (ibid. : 99).

Selon la lettre du $1^{\text {er }}$ avril 1717 adressée à Pope, elle a commencé à étudier le turc (« you see I am pretty far gone in Oriental learning; and, to say truth, I study very hard", ibid.:152); elle traduit des vers en anglais et souligne le génie différent des deux langues:

I cannot determine [...] how well I have succeded in the translation, neither do I think our English proper to express such violence of passion, which is very seldom felt amongst us. We want also those compound words, which are very frequent and strong in the Turkish language (idem).

La compétence en langue turque ${ }^{9}$ lui permettra finalement d'accéder à la connaissance directe de l'autre, notamment grâce à la rencontre avec la sultane Hafiten et à la seconde visite à Fatima, dont elle rapporte directement les mots en traduction :

I did not omit this opportunity of learning all that I possibly could of the serraglio, which is so entirely unknown amongst us. [...] She [Hafiten] seems very fond of the discourse. «My past happiness, said she, appears a dream to me [...] » There was no affectation in these words (ibid. : 217-219).

I spent the afternoon in her [de Fatima] conversation, with the greatest pleasure in the world [...] now that I understand her language, I find her wit as agreeable as her beauty. She is very curious after the manners of other countries (ibid. : 223). son ipséité (Raus 2006) grâce à la transcription des mots de l'autre, les langues 
européennes ont tout d'abord une fonction instrumentale à l'intérieur des Lettres turques. La connaissance de l'autrichien permet à Lady Mary de suivre une comédie à Vienne (ibid. : 25) ; le manque de compétence en allemand, par contre, ne lui permet pas de faire sa dévotion dans une église à Hanovre (ibid.: 69). D'ailleurs, précisons-le, le recours aux interprètes lors de l'arrivée à la frontière turque a une fonction similaire :

We wait here till all points are adjusted, concerning our reception on the Turkish frontiers. Mr Wortley's courier, which he sent from Essek, returned this morning, with the bassa's answer in a purse of scarlet satin, which the interpreter here has translated (ibid. : 91).

Ce sera justement la rencontre avec Achmet pacha à pousser Lady Mary à aller plus loin dans la découverte de l'autre, les discours des voyageurs précédents n'ayant pas réussi à restituer l'altérité dans sa propre réalité :

He assured me [le pacha Achmet], that if I understood Arabic, I should be very well pleased with reading the alcoran, which is so far from the non sense we charge it with, that it is the purest morality, delivered in the very best language (ibid.:116).

Nous ne trouvons rien de pareil lors de l'acquisition des langues européennes. Revenons donc maintenant à celles-ci, en insistant sur le cas de la langue française.

\section{La valeur du français}

\subsection{Un simple instrument de communication?}

En tant que langue européenne, le français a tout d'abord une fonction instrumentale dans la correspondance de Lady Mary des années 1715-1720 : il s'agit de la langue qui lui sert à communiquer avec des correspondant(e)s qui ne sont pas anglophones. Avant son départ pour l'Empire ottoman, par exemple, elle correspond en français avec l'Allemande Ermengarde Melusina, Baronesse du Schulenburg (Halsband 1965 : 245). En avril 1718, peu avant sa rentrée de Constantinople, elle écrit une lettre en français à Mme de Bonnac (ibid. : 403 sq.), qui pourtant ne fait pas partie des Lettres turques publiées. Dans cette lettre, elle résume les points saillants de sa découverte de l'autre ottoman, qui se fait par la vue mais aussi par la langue étrangère ${ }^{10}$ :

Je suis fort diligente de tout voir; je parle passablement la langue, \& j'ai eu l'avantage de faire amitié avec des Dames Turques, \& de leur être agreable ; \& je puis me vanter d'être la première étrangere qui ait jamais eu ce plaisir (ibid. : 404).

Encore, il nous faut mentionner les échanges en français, durant et après le voyage en Turquie, avec Nicolas-François Raymond, conseiller au Parlement parisien et ami de l'Abbé Conti. Enfin, les lettres adressées à ce dernier, avec lequel elle correspond en français, mais dont le seul original qui nous soit parvenu dans cette langue est représenté par la lettre LVIII, qui date de février 1718. Raymond s'y réfère le $20 / 9$ avril 1718 : « Monsieur l'abbé C[onti], qui est particulierrement de mes amis, m'a confié une lettre que vous lui avez écritte de Constantinople» (Halsband 1965 : 395). La citation serait une preuve de l'authenticité de cette lettre, qui fait partie d'un groupe de 6 lettres apocryphes ajoutées plus tard aux Lettres turques. Tout comme Halsband, nous pensons que les cinq autres sont fausses (Raus 2012), et que seulement celle-ci est vraie, non seulement pour la raison que nous venons de citer, mais aussi pour deux autres raisons. La première en est que l'original en français existe sous forme imprimée (Halsband 1965 : 374); la seconde, c'est que cette lettre est la réponse de Lady Mary à une lettre de l'Abbé Conti que nous avons retrouvée à l'intérieur d'un recueil de lettres 
édité plus tard par Antoine Sérieys (1802: 163 sq.). Dans sa lettre, en effet, Conti pose des questions sur le déisme des Turcs, sur les tulipes et sur des sujets variés, questions auxquelles Lady Mary répond justement par la lettre LVIII.

L'importance de cette dernière consiste dans le fait qu'elle nous renseigne aussi et surtout quant à la maittrise que Lady Mary a de la langue française :

[...] j'ay dessein de repondre exactement à toutes vos questions, du moins si mon Français me le permet. Car comme c'est une langue que je ne sçai pas à fonds, je crains fort que je seray obligée de finir bientot, faute d'expressions. Souvenez vous donc, que j'ecris dans une langue qui m'est etrangere, \& croyez bonnement que toutes les impertinences \& les fadaizes, qui partiront de ma plume, ne viennent que de mon incapacité à pouvoir exprimer ce que je pense, mais nullement de stupidité ou d'une legereté naturelle. Ces conditions ainsi faites \& stipulées, je vous dirai d'abord que vous avez une idée juste de l'Alcoran [...] J'aurois bien d'autres particularités à vous communiquer, mais je suis au bout de mon Papier \& de mon François (Halsband 1965 : 374-378).

31 Par l'extrait cité, nous pouvons faire une série de remarques à l'égard de l'acquisition que Lady Mary a faite de la langue française. En premier lieu, nous percevons une sorte d'insécurité linguistique que Lady Mary éprouverait par rapport à une langue apprise avant tout en lisant des livres. Cela nous permettrait donc de nous interroger sur sa réelle compétence en FLE, à l'encontre de ce que soutiennent Long (2009) et Grundy (1999).

À ce sujet, il nous faut ajouter que cette insécurité se lie à un discours plus général que Lady Mary fait sur les langues dans ses Lettres turques. Dans la lettre XL, adressée à Lady Rich le 16 mars 1718, Lady Mary, se référant aux difficultés qu'elle rencontre face au multilinguisme présent en Turquie, écrit :

I am in great danger of losing my English. I find 'tis not half so easy to me to write in it, as it was a twelvemonth ago. I am forced to study for expressions, and must leave off other languages, and try to learn my mother tongue ${ }^{11}$. Human understanding is as much limited as human power, or human strength. The memory can retain but a certain number of images; and 'tis as impossible for no human creature to be perfect master of ten different languages [...] As I prefer English to all the rest, I am extremely mortified at the daily decay of it in my head, where I'll assure you (with grief of heart) it is reduced to such a small number of words, I cannot recollect any tolerable phrase to conclude my letter with (Montagu 1799 : 229-231).

33 Cela nous conduit à un deuxième constat, issu de la comparaison de l'extrait français avec cette dernière lettre : la langue exprime la pensée, celle-ci se structurant en idées dont certaines sont liées aux images stockées en mémoire. Cette conception de la langue et de la pensée baigne d'ailleurs dans la culture lockéenne du XVIII ${ }^{e}$ siècle. La volonté de bien vouloir exprimer sa pensée par le langage est le noyau commun des deux extraits, ce qui renvoie à la nécessité de bien maîtriser la langue maternelle aussi bien que la langue étrangère.

Le lien entre pensée (les idées) et mots reviendra dans l'ouvrage que Lady Mary écrit en français sur la maxime de La Rochefoucauld. Dans ce livre, en effet, elle semble anticiper la critique que les révolutionnaires français feront plus tard à l'«abus des mots ${ }^{12}$ des aristocrates en s'appuyant sur l'analogie de Condillac ${ }^{13}$ (Guilhaumou: 1989) :

Rien ne décele davantage la petitesse d'esprit, que de se laisser gouverner par les mots. Si un usage absurde, quoique fondé sur quelques raisons, a pu jetter une sorte de ridicule sur les mots de mari \& de femme, c'est que dans l'acception commune, 
celui de mari offre l'idée d'un tiran jaloux \& fâcheux, ou du moins d'un sot, dupe \& crédule ; \& celui de femme désigne un animal domestique, tracassier, capricieux \& léger, destiné à tromper \& à désoler un malheureux ami. La conduite en général des personnes mariées suffit sans doute pour justifier ces idées populaires. Mais pourquoi, comme je l'ai déjà dit, nous laisserons-nous imposer par les mots? (Montagu[e] 1768 : 65-66) considéré par Lady Mary comme " langue étrangère », c'est qu'à l'intérieur du « nous " - chrétienté, dont nous avons déjà parlé, il faut ajouter des éléments qui permettent de voir en quoi le français acquiert sa propre spécificité qui le distingue des autres langues européennes, et cela malgré la présence d'une matrice identitaire commune.

\subsection{Le rapport conflictuel avec une langue de prestige}

Nous avons dit qu'à la fin du XVII ${ }^{e}$ siècle et au tout début du XVIII ${ }^{e}$, les compétences en FLE sont une vraie marque sociale, le français étant considéré comme langue de prestige. Suso López (2005: 6) souligne que lors de l'apprentissage de cette langue, il s'agissait d'acquérir un "savoir savant", véhiculé par la cour et la mondanité des salons. La difficulté de l'apprentissage consistait aussi dans le fait que cette langue, qui s'était formalisée dans la grammaire de Port-Royal et dans des dictionnaires, présentait malgré tout des variabilités d'ordre diachronique, diatopique et diastasique.

Pour accéder à ce "savoir savant ", Lady Mary lisait, entre autres, les romans de l'amour précieux. Ces lectures continueront aussi lors de son retour de Constantinople, en se faisant plus variées :

Lady Mary gathered French books as regularly as English one. Here the "canon » was represented by the CEuvres of J.B. Rousseau, 1723, and of Marie-Catherine Desjardins, Madame de Villedieu, 1721. [...] She acquired personal history and fiction (two virtually indistinguishable genres) about the French seventeenth century. She acquired travel writings: Maximilien Misson on Italy. The Enlightenment supplied a volume of works by thinkers and philosophers which, although edited by Pierre Desmaiseaux, was the brainchild of Conti, and Lettres galantes et philosophiques, 1721, by Rémond de Saint-Mard [...] This small segment of Lady Mary's book acquisition reflects consistent tastes. She loved poetry, drama, fiction, and knotty thought. She felt as much at home in French as English (Grundy 1999: 195-196).

Les gallicismes, plus ou moins intégrés, qui sont présents dans les Lettres turques nous montrent que le français était tout d'abord la langue des salons et des différents types d'amour qui animaient les conversations à la mode de l'époque : "prudery, galantry, coquette, prude, libertine, belles passions, ruelle» sont des emprunts qui renvoient justement aux différentes conceptions de l'amour mondain rendu célèbre par les Français ${ }^{14}$. D'ailleurs, il s'agit d'« une Nation qui se pique de surpasser en politesse le reste du monde, \& qui depuis longtemps donne des règles de galanterie à toute l'Europe » (Montagu[e] $1799: 61$ ).

Parmi les gallicismes, il y en a un qui nous renseigne sur l'influence que cette langueculture exerçait sur l'imaginaire de Lady Mary, puisqu'il s'agit d'un emprunt de prestige qui donc, à la différence des autres, n'est pas nécessaire dans la mesure où l'équivalent indigène lui préexiste. Il s'agit de l'expression "voyage-writer $\aleph^{15}$, utilisée dans les Lettres turques au lieu de " travel-writer ${ }^{16}$. En effet, quand Lady Mary parle des voyageurs, il faut y compter également les Français qui ont été les plus nombreux à 
partir pour l'Empire ottoman jusqu'à la première moitié du XVIII ${ }^{\mathrm{e}}$ siècle ${ }^{17}$. C'est pour cela qu'outre des voyageurs anglais comme Paul Rycaut, Aaron Hill et George Sandys, Lady Mary cite aussi des voyageurs français, comme Jean Dumont et un auteur français qu'elle préfère ne pas citer explicitement ${ }^{18}$.

Lors de ses lectures en français, Lady Mary a donc affaire à deux mémoires discursives: la première est celle que les écrivain(e)s de l'amour mondain ont construite autour de la galanterie; la seconde consiste dans la mémoire que les voyageurs ont tissée autour de la Turquie avant le départ de la Lady anglaise. Bien que fascinée par les deux, Lady Mary finit en fait par s'y opposer.

Pour ce qui est de l'amour, elle tournera en ridicule la fausseté des dames françaises lors de son séjour à Paris (Lettre LXIX à Lady Rich, notamment p. 305-306) et finira par se positionner clairement contre la galanterie dans son discours sur la maxime de La Rochefoucauld :

une passion, qui est le mobile de tous les beaux exploits de la galanterie moderne, \& qui n'a d'autre but que d'appaiser ses ardeurs par la jouissance de l'objet qu'on trouve aimable; passion fondée sur l'injustice, \& qui traine à sa suite le crime, le remords, la jalousie \& le mépris. Une affection semblable pourroit-elle être délicieuse pour une ame vertueuse? Cependant tel est le principe de tous ces engagements illustres de galanterie (Montagu[e] $1768: 64$ ).

Pour ce qui est de la tradition naissante de la littérature de voyage, la critique qu'elle fait aux mensonges des voyageurs concerne bien évidemment les Français aussi, comme on peut voir par ce commentaire : «I see you have taken your ideas of Turkey, from that worthy author Dumont, who has wrote with equal ignorance and confidence » (Montagu 1799 : 202).

Nous comprenons que ce qui pousse Lady Mary à s'opposer au génie français ${ }^{19}$, qui se dessine par leur langue, c'est le manque de vérité et de transparence.

\section{Conclusion}

À la fin de cet excursus, nous pouvons affirmer que Lady Mary attribue aux langues au moins quatre valeurs épistémologiques :

1. en tant que voyageuse, elle leur donne une valeur instrumentale : la langue permet en ce sens d'acquérir un "savoir faire » spécifique (écrire à ses correspondants, profiter des lieux de visite) ;

2. en tant qu'aristocrate, elle attribue une valeur de prestige à la langue française et essaie d'acquérir un « savoir savant » par cette langue via ses lectures ;

3. en tant que (proto-)ethnologue, elle fait acquérir à la langue-autre, dans ce cas le turc, la valeur de la découverte qui lui permet de « savoir connaitre » l'altérité ;

4. en tant que (proto-)philosophe, elle attribue une valeur herméneutique aux langues, qui permettent de "savoir exprimer » la pensée. Pour cela elle éprouve un sentiment d'insécurité linguistique, notamment par rapport au français, qui reste, malgré tout, une langue étrangère.

À la croisée entre institutionnalisation et disciplinarisation, Lady Mary donne au français une valeur de prestige aussi bien qu'une valeur instrumentale et herméneutique à la fois, tout en entretenant avec cette langue un rapport conflictuel 
lié à l'image qu'elle en avait par ses lectures et à la désillusion de la réalité lors de son séjour en France.

\section{BIBLIOGRAPHIE}

\section{Sources}

HALSBAND, Robert (éd.) (1965). The complete letters of Lady Mary Wortley Montagu.Oxford : OxfordClarendon Press, vol. 1 (1708-1720).

MONTAGU, Lady Mary (1799). Letters of the Right Honorable Lady Mary Wortley Montague, Written during her Travels in Europe, Asia and Africa.Berlin : Mylius.

MONTAGU[E], Lady Mary (1768). Lettres de Milady Worthley Montague, écrites pendant ses voyages en diverses parties du Monde. Troisième partie pour servir de Supplément aux deux premières. ParisLondres : veuve Duchesne.

SÉRIEYS, Antoine (éd.) (1802). Lettres inédites d'Henri IV et de plusieurs personnages célèbres, tels que Fléchier, La Rochefoucault, Voltaire, le comte de Caylus, Anquetil-Duperron, etc. imprimées sur les originaux. Paris : H. Tardieu.

Études

ARGAUD, Évelyne (2009). «Les enjeux des représentations des langues savantes et vulgaires en France et en Europe au XVI ${ }^{\mathrm{e}}$ et XVII ${ }^{\mathrm{e}}$ siècles ». Documents pour l'Histoire du Français Langue Étrangère ou Seconde, 43, 9-24. [http://dhfles.revues.org/815]

AUROUX, Sylvain (1993). Histoire des idées linguistiques. Liège : Mardaga.

BRANCA-ROSOFF, Sonia (éd.) (2001). L'institution des langues. Paris : MSH.

FERNÁNDEZ FRAILE, Maria Eugenia (2012). « Avec quels ouvrages les femmes apprenaient (et enseignaient) les langues? Bibliographie raisonnée (XV $\mathrm{XV}^{\mathrm{e}}-\mathrm{XIX}{ }^{\mathrm{e}}$ siècle) ». Documents pour l'Histoire du Français Langue Étrangère ou Seconde, 47/48, 45-64.

GRUNDY, Isobel (1999). Lady Mary Wortley Montagu. Oxford : Oxford-Clarendon Press.

GUILHAUMOU, Jacques (1989). La langue politique et la Révolution française. Paris : Klincksieck.

KAHANE, Henry (1986). « A Typology of the Prestige Language ». Language, 62, 495-508.

KIBBEE, Douglas (2001). « From Holyband to Mauger : Teaching French in $17^{\text {th }}$ century in England ». In Jan De Clerq, Nico Lioce, Pierre Swiggers, Grammaire et enseignement du français. 1500-1700, Louvain : Peeters, 179-196.

KIBBEE, Douglas (1991). For to speke Frenche Trewely. The French Language in England, 1000-1600 : its status, description and instruction. Amsterdam, Philadelphia : Benjamin Press.

LONG, Lynne (2009). «Lady Mary translates Marivaux. A Female Perspective ». Palimpsestes, 22, 129-147.

[http://palimpsestes.revues.org/198] 
PASTON, George (1907). Lady Mary Wortley Montagu and her times. London, New York : G.P. Putnam's Sons.

PELOUS, Jean-Michel (1980). Amour précieux, amour galant (1654-1675). Essai sur la représentation de l'amour dans la littérature et la société mondaine. Paris : Klincksieck.

RAUS, Rachele (2012). « The Letters of Lady Mary Wortley Montagu in France between ideological turn and women's writing ». In Susan Pickford \& Alison Martin, Travel narratives in translation, 1750-1850 : Nationalism, Ideology, Gender. London : Routledge, 157-180.

RAUS, Rachele (2006). « Les Lettres de Lady Mary Montague : réception en France d'une écriture féminine ».Publif@rum, 3, 2006.

[URL : http://farum.it/publifarumv/n/03/raus.php]

RAUS, Rachele (2001). « La prise de parole du Turc dans la littérature de voyage française aux $\mathrm{XV}^{\mathrm{e}}-\mathrm{XVI}^{\mathrm{e}}$ siècles : du silence fondateur à la suppression des stratégies de silenciement ». In Mariagrazia Margarito, Enrica Galazzi, Monique Lebhar Politi, Oralité dans la parole et dans l'écriture. Torino : Cortina, 199-214.

RAUS, Rachele (2000). Semantica ed analisi del discorso : il lemma Turc dal XVI alla prima metà del XIX secolo : Thèse de doctorat présentée à l'Université de Trieste (non publiée).

SALMON, Vivian (1994). « Women and the study of language in sixteenth and seventeenth century England ». HEL, 16/2, 95-119.

SUSO LÓPEZ, Javier (2012). « Le rôle des Grammaires destinées aux dames dans la disciplinarisation du français (XVIII ${ }^{\mathrm{e}}$ siècle) ». Documents pour l'Histoire du Français Langue Étrangère ou Seconde, 47/48, 65-80.

SUSO LÓPEZ, Javier (2005). « Le rôle des 'maîtres' dans la construction du français langue étrangère comme discipline scolaire ». Documents pour l'Histoire du Français Langue Étrangère ou Seconde, 33/34, 94-109. [URL : http://dhfles.revues.org/1702]

\section{NOTES}

1. Nous avons consulté l'édition anglaise de 1799 qui reproduit fidèlement l'original de 1763 et dont nous conservons l'orthographe.

2. Il est utile de préciser qu'à cette époque « a few women demonstrated their linguistic talents by writing in a foreign language » (Salmon $1994: 107)$.

3. Rappelons que la cour anglaise était par ailleurs étroitement liée à la France grâce au mariage entre Charles I et Henriette de France et à la liaison entre Charles II et Louise de Kerovalle (Kibbee $2001: 180$ ).

4. Mary Pierrepont (1689-1762) se marie à Edward Wortley Montagu (Worthley Montague dans la graphie française) en 1712 .

5. Suso López précise à ce sujet que « dans le domaine linguistique, l'éducation latine n'est pas conseillée [aux femmes] » (2012:66).

6. Plus tard, son mari Edward Wortley Montagu l'incitera à ce genre d'apprentissage, au point qu'elle l'appellera son « maître ».

7. D'ailleurs, la cour anglaise elle-même est définie comme "the fairest in Christendom» (Montagu 1799 : 151).

8. L'Abbé Conti, sur lequel nous reviendrons, était un savant italien avec lequel Lady Mary échangeait des lettres en français. 
9. Dans la lettre XXXVI du 17 juin 1717, elle confie à Pope d'y travailler assidûment tous les jeudis (Montagu 1799 : 199).

10. À l'égard de la langue turque, Lady Mary utilise des stratégies similaires à celles que Guillaume Postel avait employées au XVI ${ }^{\mathrm{e}}$ siècle (Raus 2001). Par exemple, les listes plurilingues des mots étrangers sont présentes ici de manière indirecte, sous forme d'emprunts turcs glosés lors de la description des objets-autres.

11. Dans la lettre XXXVI du 17 juin 1717, elle dit à Pope qu'elle dédie tous les mardis à sa langue maternelle (Montagu 1799 : 199).

12. Pourtant, dans le cas de Lady Mary l'abus ne peut absolument pas être reconduit à l'aristocratie. C'est plutôt le registre "vulgaire» qui est mis en question dans les Lettres turques (Montagu 1799 : 144, 202).

13. Remarquons, entre autres, que Condillac a joué un rôle fondamental dans la vulgarisation des travaux de Locke en France.

14. Jean-Michel Pelous précise que "de cette époque [aux alentours de 1650] date le fameux triangle 'prude, précieuse, coquette' qui permet de décrire avec de très subtiles nuances les diverses formes que peut revêtir la rébellion contre l'amour » (Pelous 1980 : 246).

15. Montagu 1799 : 164, 258. Le mot « voyage » tout court est également présent (Montagu 1799: 202, 263).

16. Cependant soulignons que «traveller » est également utilisé.

17. Le nombre élevé de voyageurs français en Turquie se doit au rapprochement diplomatique inauguré par le traité bilatéral signé entre François ${ }^{\mathrm{er}}$ et Soliman le Magnifique au XVI ${ }^{\mathrm{e}}$ siècle (Raus 2000).

18. "A certain French author says Constantinople is twice as big as Paris » (Montagu 1799 : 192).

19. D'ailleurs, la critique aux dames françaises dans les Lettres turques déclencha l'aversion des celles-ci envers son livre (voir Raus 2012). Nicolas-François Rémond en parle dans l'une des lettres adressée à Lady Mary : «Me refuserez vous toujours vos conseils avec opiniatreté et parce que vous haissez (à ce que tout le monde dit à Paris) la France et tous les Francois ?» (Halsband $1965: 451)$.

\section{RÉSUMÉS}

En tant qu'aristocrate anglaise, Lady Mary Worthley Montague eut l'occasion de voyager à côté de son mari Edward, lors de l'Ambassade de ce dernier à la Porte ottomane en 1717. Elle profita de ce séjour au Levant pour apprendre la langue turque et s'entretenir avec des musulman(e)s. Elle visita également l'Europe et décrivit, entre autres, les mœurs de la cour française. À partir de son voyage, elle rédigea ses célèbres Turkish Embassy Letters, traduites en français dès 1763. L'analyse des lettres permet de retracer la valeur épistémologique que les langues étrangères, notamment le français, avaient pour Lady Mary. Si l'apprentissage de la langue turque se lie à la découverte de l'autre-musulman, la connaissance et l'utilisation du français semblent avoir une fonction absolument différente. La Lettre LVIII (Halsband 1965), dont l'original est en français, fait mieux comprendre la vraie valeur de cette langue de «prestige » (Kahane 1986).

As an English aristocratic woman, Lady Mary Wortley Montagu travelled with her husband Edward to the Ottoman Porte in 1717 and described her journey in the famous Turkish Embassy Letters, translated in French since 1763. She could learn Turkish language and spoke with some 
Muslims, especially with women. She could also visit European courts, among them the French court. The analysis of her Turkish Letters let us establish what was the epistemological value Lady Mary gave to foreign languages, namely to the French one. If the Turkish language was used to discover alterity, the role of French was completely different. The Letter $n^{\circ} 58$ (Halsband 1965), written in French, demonstrates that this «prestigious» language (Kahane 1986) had a very particular value.

\section{INDEX}

Mots-clés : apprentissage des langues étrangères, français langue étrangère, XVIIe-XVIIIe siècles, Lady Mary Worthley Montague, français langue de prestige

Keywords : learning foreign languages, French as foreign language, 17th-18th centuries, Lady Mary Wortley Montagu, French as prestigious language

\section{AUTEUR}

\section{RACHELE RAUS}

Université de Turin

racheleraus@libero.it 